\title{
Free Grafting of the Extensor Digitorum Longus Muscle in the Rat after Marcaine Pretreatment
}

\author{
Bruce M. Carlson ${ }^{1}$ and Ernest Gutmann \\ Department of Anatomy, University of Michigan, Ann Arbor, Michigan 48109, and \\ Institute of Physiology, CSAV, Budějovická 1083, Praha 4,KRC, Czechoslovakia
}

Reccived April 30, 1976

\begin{abstract}
Normal or predenervated extensor digitorum longus muscles in the rat were given one or two preinjections of a solution of Marcaine and hyaluronidase and then freely autografted into normally innervated or denervated limbs. This procedure caused the sarcolysis of virtually all the original muscle fibers within the graft, followed by a massive regenerative response that restored the normal number of muscle fibers to the grafted muscle. Contraction times of grafts were very slow when contractile responses were first elicited by direct stimulation of the muscles. In normally innervated limbs, contraction speeded up progressively until early in the second month when the speeds approached those of the normal muscle. Grafts placed into denervated legs followed a similar pattern of development of contractile properties during the first 3 weeks, but after that time the nerveless grafts contracted progressively more slowly than their reinnervated counterparts. Mature grafts developed a histochemically heterogeneous pattern of muscle fiber types (Types I and II) after staining for adenosine triphosphatase and succinic dehydrogenase activity.
\end{abstract}

\section{INTRODUCTION}

Although the authors have studied a number of experimental models by which the development of contractile properties of regenerating muscles in the rat could be measured, none of these has proved completely satisfactory. The minced gastrocnemius provides a pure population of regenerating muscle fibers, but the architectural disruption of the muscle due to the mincing and the large amount of connective tissue that forms in regenerating minced muscle in the rat make this less than an ideal physiological model (4). In addition the gastrocnemius-plantaris complex in the rat is

i Supported by grants from the Muscular Dystrophy Association and the National Institutes of Health and a scientific exchange between the Academies of Sciences of the United States and Czechoslovakia. 
mixed with respect to fiber type, with a greater proportion of slow muscle fibers than that found in some other fast twitch muscles. The slow soleus and fast extensor digitorum longus mucles have proved especially useful for physiological studies, but in the rat these muscles do not regenerate well after mincing. Free grafting of these muscles, both in the normal and predenervated condition, provides a substantial population of regenerating muscle fibers of a more uniform fast or slow type, but analysis of developmental gradients of contractile properties is complicated by the survival of original muscle fibers at the periphery of the graft $(5,6)$. Grafting of a transversely sliced muscle eliminates surviving muscle fibers, but the disruption of internal architecture of the muscle by the slicing interferes with the full development of fuction (9).

The local anesthetic agent, Marcaine, produces a rapid degeneration followed by regeneration of muscle fibers following injection $(1,11)$. Recently a technique combining the injection of Marcaine plus hyaluronidase into the muscle (10) with free grafting has been shown to eliminate virtually all surviving muscle fibers and to allow the regeneration of a normal number of fibers in the intact extensor digitorum longus muscle (3). In the present study, we have used the Marcaine model to follow the development of contractile properties of a pure population of regenerating nnuscle fibers in the extensor digitorum longus muscle in both normally innervated and denervated limbs in the rat.

\section{MATERIALS AND METHODS}

This study was conducted on 2- to 3-month-old male Sprague-Dawley and Wistar rats. Forty-five animals were used for a preliminary histological survey of the course of regeneration in Marcaine-treated free muscle grafts, and approximately 70 rats were used for physiological and histochemical studies. Transplanted muscles were studied from 1 to 60 days after grafting.

Muscles were treated with Marcaine in two ways, both of which produced virtually identical results. Because of the recent report of HallCraggs (10), saline solutions of $0.75 \%$ Marcaine (Winthrop) were combined with hyaluronidase-300 (beef testis, ICN Pharmaceuticals). One milligram of hyaluronidase (300 USP or international units/mg) was added to $10 \mathrm{ml}$ Marcaine solution. One-half the nutscles in the histological series and all of those in the physiological and histochemical series were given two treatments with Marcaine-one injection 2 days before grafting (preinjection) and one at the time of grafting. The remaining muscles were treated only at the time of grafting. The preinjection technique consisted of surgically exposing the muscle and injecting it with as much Marcaine solution as it would hold (about $0.2 \mathrm{ml}$ for a normal muscle and $0.1 \mathrm{ml}$ for a 15-day predenervated muscle). At the time of grafting, all 
muscles to be grafted, whether preinjected or not, were removed from the leg, injected with as much Marcaine solution as they could hold, and then immersed in Marcaine solution for $10 \mathrm{~min}$ before being grafted into the contralateral leg.

All rats were operated on under ether anesthesia. Free grafting of the extensor digitorum longus muscle was done in the manner previously reported $(5,6)$. The muscle to be grafted was removed, injected and soaked with Marcaine, and grafted in place of the contralateral extensor digitorum muscle. Each end of the graft was sutured to the appropriate tendon stump. The stump of the motor nerve was allowed to lie alongside the grafted muscle without being fixed to the graft. Vascular reanastomoses were not made. Denervation of rat legs was accomplished by transecting the sciatic nerve high in the thigh and tying the proximal stump of the nerve.

For contractile analysis, grafts were removed from animals $4,7,14,30$, and 60 days after transplantation. They were placed in a chamber at $36^{\circ} \mathrm{C}$ and containing an oxygenated culture medium $(149.8 \mathrm{~mm} \mathrm{Na}+5.0 \mathrm{~mm} \mathrm{~K}$, $2.0 \mathrm{mM} \mathrm{Ca}^{2+}, 148.0 \mathrm{~mm} \mathrm{Cl}-12.0 \mathrm{~mm} \mathrm{HCO}_{3}^{-}, 1.0 \mathrm{mM} \mathrm{H}_{2} \mathrm{PO}_{4}$, and 11.0 $\mathrm{mm}$ glucose), $\mathrm{pH} 7.2$, to which tubocurarine $(0.01 \mathrm{M})$ had been added. After a 10-min equilibration period, the optimal resting tension for isometric recording was determined by direct stimulation of the muscles with platinum electrodes. The following contractile properties were recorded with the automatic analyzer of muscle contraction properties that was used in our previous studies: twitch and tetanic tension, latency period (stimulus artifact to first mechanical response), contraction time (time to peak tension), half relaxation time (from peak to one-half amplitude of the twitch), and time parameter of both twitch and tentanic tension development (14).

Contractile properties were determined on four series of grafts. In all series the muscles were preinjected with Marcaine 2 days before grafting and injected again at the time of grafting.

Series I: 14-day predenervated muscle grafted into the contralateral normal limb.

Series II: 14-day predenervated muscle grafted into the contralateral denervated limb.

Series III: normal muscle grafted into the contralateral normal limb.

Series $I V$ : normal muscle grafted into the contralateral denervated limb.

Long-term grafts ( 30 and 60 days) that had been used for contractile analysis were frozen in liquid nitrogen, and frozen sections were stained for both succinic dehydrogenase (12) and myosin adenosine triphophatase [ATPase $(8,13)]$. Short-term grafts from the contractile series, as well as all grafts in the histological series, were fixed in Bouin's solution, sectioned at $7 \mu \mathrm{m}$ and stained with Ehrlich's hematoxylin and eosin. 


\section{RESULTS}

\section{Morphology}

The developmental histology of normal muscle grafts that had been injected with Marcaine at the time of grafting was similar in most respects to that which has been already noted in grafts of normal muscles (5). During the first few days, most of the graft was ischemic. Only at the outermost edges were cellular reactions seen. A thin zone of degenerating muscle fibers had become established around much of the periphery. By 2 days there were already faint differences in staining between the major-

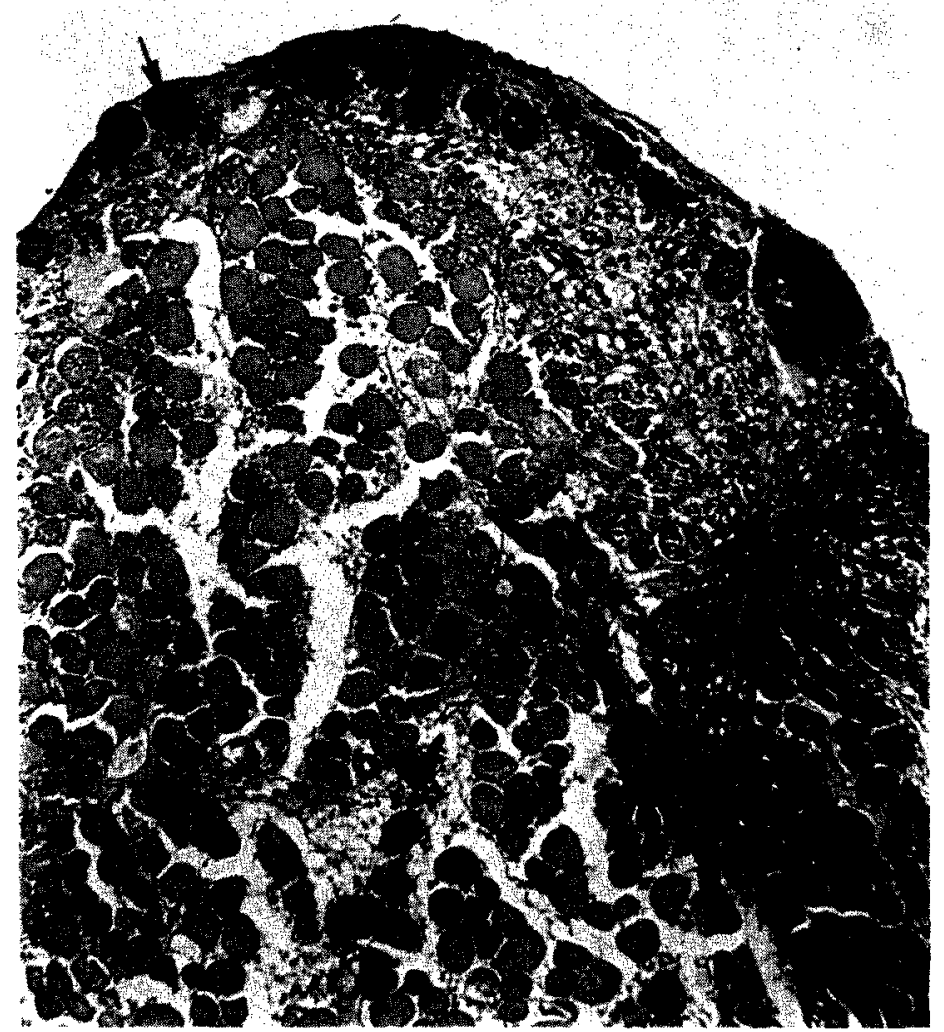

FIG. 1. Cross section through 2-day graft of muscle that had been treated with Marcaine at the time of grafting. Scattered intact peripheral muscle fibers (arrow) stain more darkly than those of the ischemic central mass. At this stage it is still too early to know whether or not the peripheral muscle fibers will survive. Several of them have highly vacuolated sarcoplasm. Between the peripheral intaot fibers and the central ischemic region is a thin zone where sarcolysis of original muscle fibers is taking place and where myoblastic cells are appearing. Hematoxylin and eosin, $\times 90$, 
ity of surviving muscle fibers within the zone of degeneration and occasional intact muscle fibers peripheral to it (Fig. 1). The latter stained somewhat more darkly with eosin, and it is possible that small numbers of these fibers do survive the grafting procedure, although in Marcainetreated muscle grafts only occasional original muscle fibers survive (3). At 4 to 5 days the grafts consisted of a peripheral area containing numerous myotubes with few or no surviving muscle fibers, and a smaller central area in which the ischemic original muscle fibers were degenerating (Fig. 2). Early in the second week the grafts contained a homogenous population of late myotubes and early cross-striated muscle fibers (Fig. 3).

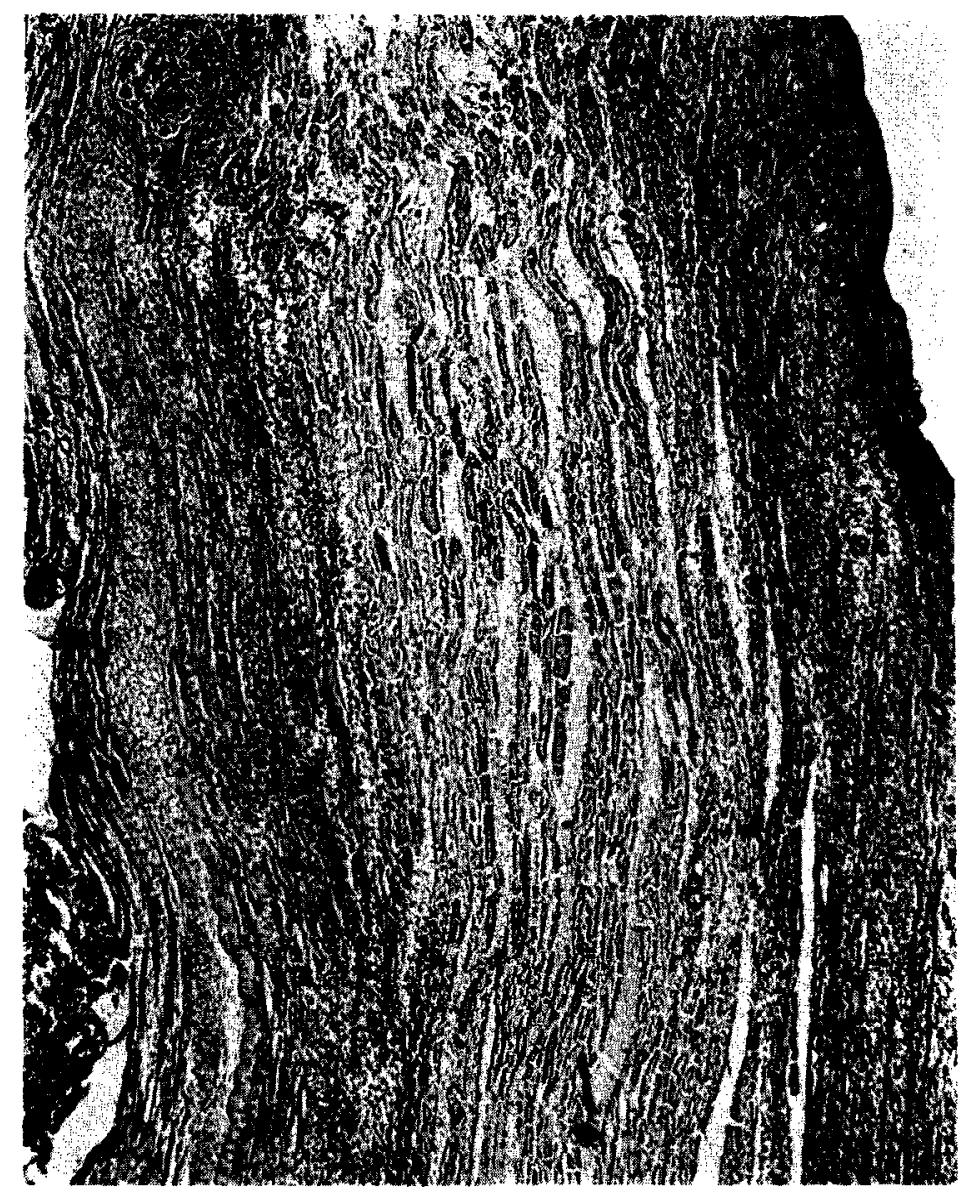

Fig. 2. Longitudinal section through a 5-day graft of Marcaine-treated muscle. A peripheral zone of regeneration, containing numerous myotubes, surrounds a progressively smaller central zone of lightly stained degenerating original muscle fibers. Hẹmatoxylin and eosin, $\times 40$. 


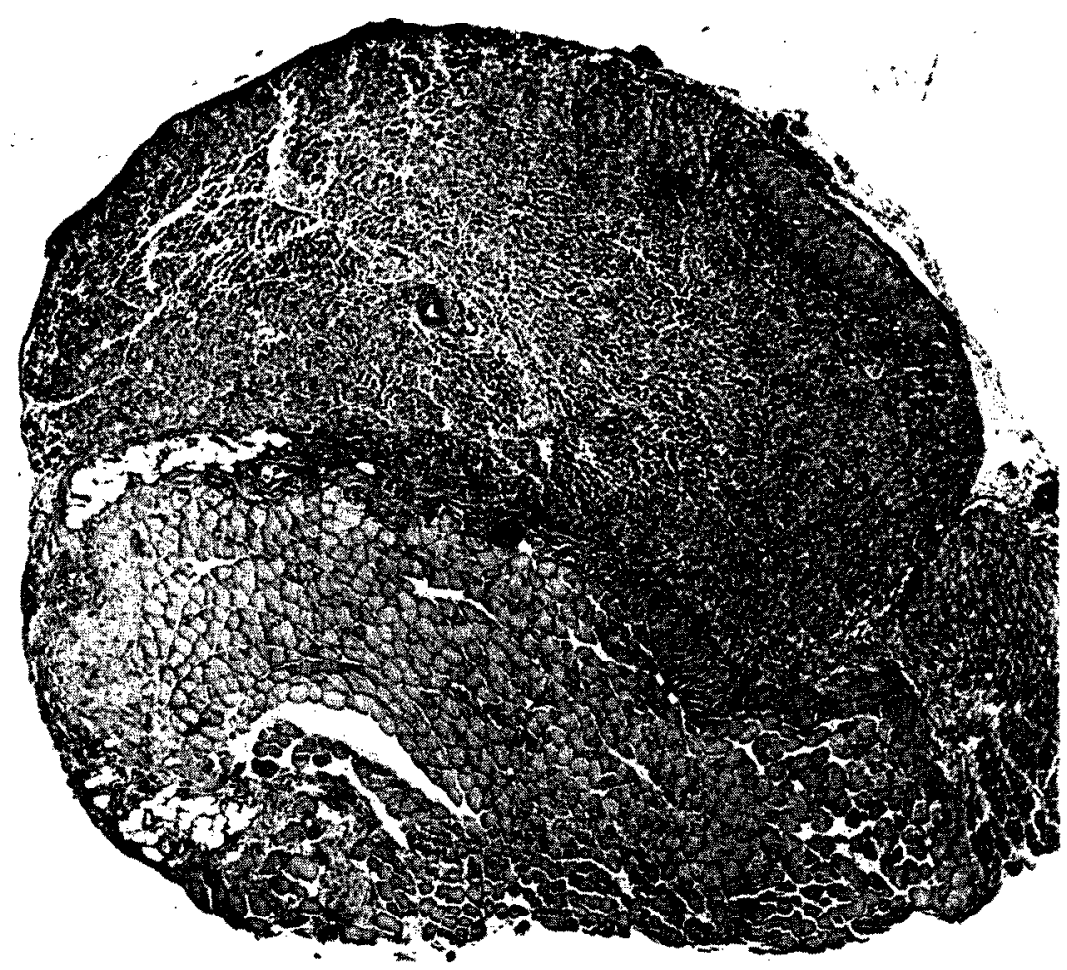

FIG. 3. Cross section through a 10-day graft of Marcaine-treated muscle (above) next to part of the adjacent normal tibialis anterior (below). This section shows the great difference between the diameter of normal and regenerating muscle fibers. Hematoxylin and eosin, $\times 38$.

The central region of ischemic muscle had disappeared. After this time the muscle fibers of the grafts gradually matured, and by 60 days they had become stabilized at about the same number of muscle fibers found in the normal extensor digitorum longus muscle.

Normal muscles which had received an injection of Marcaine 2 days before grafting reacted somewhat differently from the above group, but after the second week there was no identifiable difference between them. The major differences between muscles in this group and those which had been injected only at the time of grafting were a more rapid disappearance of old muscle fibers from the center of the graft and a slight (1-to-2-day) acceleration of the early stages of differentiation (e.g., myotube formation) of the regenerating muscle fibers. By 4 days the preinjected grafts were filled with myotubes and immature connective tissue. These grafts demonstrated a less pronounced radial gradient of differentiation and therefore a somewhat more synchronous population of early regenerating muscle cells than did muscles that were injected only 
at the time of grafting. Muscles that had been predenervated 14 days and also preinjected with Marcaine (contractile Series I and II) were similar in early histology to grafts that had been preinjected with Marcaine only. In all groups, more mature grafts (more than 10 days) in innervated limbs were virtually indistinguishable from one another after the second week.

\section{Contractile Properties}

Control Series. In each of four rats one extensor digitorum longus muscle was predenervated 14 days and then injected with Marcaine. Two days later the muscles were subjected to contractile analysis. The purpose of this control was to determine whether or not any functioning muscle was left after a single injection of Marcaine and if so, what were the contractile characteristics of the muscles at the time of transplantation. It was obvious from the twitch and tetanic tensions alone $(0.84 \pm 0.30 \mathrm{~g}$ and $1.76 \pm$ $0.76 \mathrm{~g}$, respectively) that the muscles were severely damaged. These values are approximately $10 \%$ of the values for normal muscles of the same age. It is thus apparent that some muscle fibers had escaped total damage from the injected Marcaine. The contraction times were slowed (latency period: $3.2 \pm 0.06 \mathrm{msec}$; contraction time : $17.80 \pm 0.36 \mathrm{msec}$; half relaxation time : $26.17 \pm 2.37 \mathrm{msec}$ ), in the manner characteristic of denervated muscle.

Series 1 . The predenervated, Marcaine-treated grafts placed into normal legs provided the most satisfactory developmental gradient of contractile properties so far obtained on regenerating muscle (Table 1). From very low values at 4 days, both twitch and tetanic tensions rose to about $50 \%$ of the values of control muscles of the same age by 69 days postgrafting. All contractile times measured were very slow in early grafts and progressively speed up to normal values by 60 days.

Series II. The predenervated Marcaine-treated grafts placed into denervated legs followed a developmental course similar to th grafts in Series I through the first 2 weeks (Table 1), i.e., they showed a temporary shortening of contraction time and other parameters. By 30 days, the contraction times of the noninnervated grafts were somewhat slower than those of innervated grafts, but the contractile strength remained high. The 60-day, noninnervated grafts not only contracted much more slowly than innervated grafts but in fact regained the contraction properties observed in the earliest phases of muscle development. Their contractile strength, however, was very low.

Series $I I I$ and $I V$. Grafts of normally innervated Marcaine-treated muscle differed from those of predenervated, Marcaine-treated muscle (Series I and II) primarily during the first week after transplantation. At 4 days, some of the grafts were not yet able to contract, whereas in 


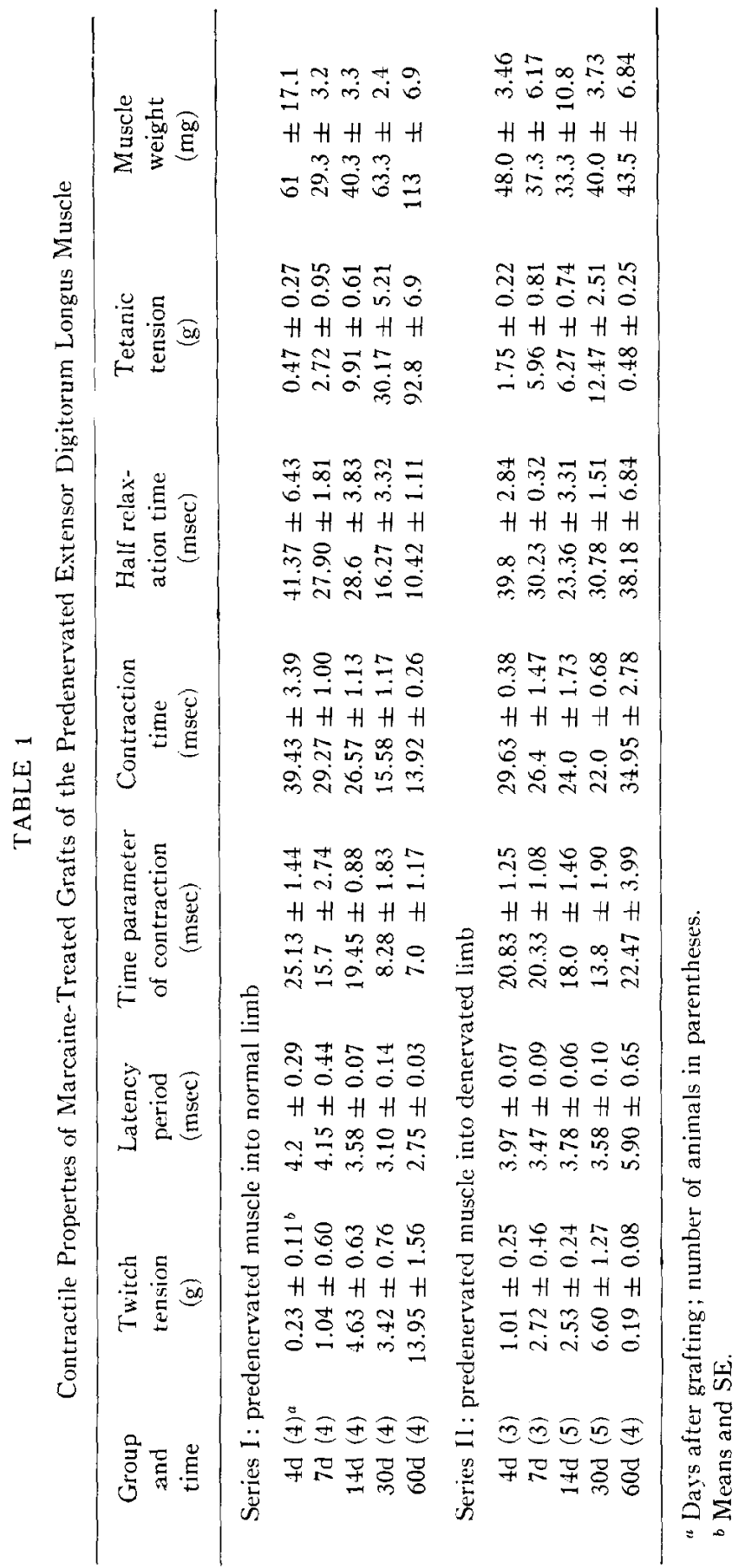







others very weak contractions could be elicited only after a stimulus with a duration of $5 \mathrm{msec}$ (Table 2). By 7 days there was already relatively little difference between most contractile properties of normal (Series III) and predenervated (Series I) grafts. As in Series II, the contraction times of 30-day noninnervated grafts in Series IV had become slower than those of innervated grafts (Series III).

\section{Histochemistry}

As was the case in our previous studies (5) long-term (60 day) grafts possess histochemically differentiated muscle fibers (Types I and II) with respect to both succinic dehydrogenase and ATPase staining. Fiber-type gruuping was again the rule in innervated grafts. In contrast, muscle fibers of grafts placed into denervated legs were atrophic and not histochemically differentiated.

\section{DISCUSSION}

This report presents a method whereby a large and developmentally uniform population of regenerating skeletal muscle fibers can be obtained in the virtual absence of contaminating orginial nuscle fibers. This combination had not previously been obtained in regenerating rat muscles. As a result of this experimental model, it is now possible to define the time course of development of contractile properties in a regenerating fast muscle (Table 1, Series I).

It is now becoming clear that in small muscles, at least, the end result of free grafting is very similar despite widely differing pretreatment. Carlson (3) has recently shown that long-term muscle grafts that had been treated in four different ways (no treatment, 14-day predenervation, and one or two preinjections with Marcaine) contained the normal number of muscle fibers for the extensor digitorum longus. Likewise, the contraction times of mature muscle grafts approach those of the normal muscle regardless of pretreatment.

The major differences between muscle grafts pretreated in different ways are seen during the first week after grafting. Various numbers of peripherally located surviving original muscle fibers are seen in grafts of normal or predenervated muscles, whereas few remain in Marcainetreated grafts. After 2 weeks any surviving muscle fibers are quantitatively overwhelmed by the large numbers of regenerating muscle fibers.

The two parameters of early postgrafting development that are most noticeably affected by the differing modes of pretreatment are the rate of disappearance of sarcoplasm of the ischemic original muscle fibers and the time of appearance and numbers of early stages in the redifferentiation of regenerating muscle fibers. In grafts of the normal extensor digitorum 
longus muscle, a progressively shrinking central core of degenerating old muscle fibers persists for most of the first postoperative week (5). Muscles injected once with Marcaine at the time of grafting lose their central core of old muscle slightly more rapidly. Muscles either pretreated with Marcaine or predenervated have lost virtually all original ischemic muscle by the third day after grafting.

The rate and amount of early muscle fiber differentiation is proportional to the disappearance of old muscle fibers. Thus in these experiments regenerating muscle fibers capable of contraction were uniformly present in 4-day grafts of predenervated Marcaine-treated muscle whereas at that time only the first traces of contractile activity could be seen in muscle fibers of normal muscles that had been treated with Marcaine in a similar way. It appears likely that a 2-day preinjection with Marcaine and, to a greater extent, predenervation stimulate the process of activation of potentially myogenic cells or nuclei to begin before the muscle has been grafted, thus giving some of the regenerating muscle fibers a head start. In his studies in vitro, Bischoff (2) has similarly noted a 1- to 2-day lag period between the application of a regenerative stimulus and the morphological activation and/or mitosis of myogenic cells. The nature of the activating stimulus remains wholly unknown.

The differences in both contractile and histochemical properties between grafts in normal and denervated legs confirm the findings of our previous study (7), that contraction times shorten in the early phases of regeneration despite the absence of a motor nerve supply. Because the major denervation effects are observed at 30 and 60 days, the Marcaine-injection model of muscle regeneration does not appear to be of any great advantage over the simpler model of grafting a normal muscle into denervated leg (5-7) except that the present findings do eliminate the possibility of our previous results being influenced by surviving muscle fibers even in long-term grafts.

It now appears that the most uniform experimental model for studying physiological and certain biochemical properties of a regenerating muscle is the free grafting of a muscle that has been injected with Marcaine before grafting. This procedure results in a homogeneous initial population of muscle cells. For most purposes only a single injection of Marcaine at the time of grafting is sufficient to produce the desired result.

\section{REFERENCES}

1. Benoit, P. W., and W. D. Belt. 1970. Destruction and regeneration of skeletal muscle after treatment with a local anaesthetic, bupivacaine (Marcaine). J. Anat. 107 : $547-556$.

2. Bischoff, R. 1975. Regeneration of single skeletal muscle fibers in vitro. Anat. Rec. $182: 215-236$. 
3. Cardson, B. M. 1976. A quantitative study of muscle fiber survival and regeneration in normal, pre-denervated and Marcaine-treated free muscle grafts in the rat. Exp. Neurol. 52: 421-432.

4. Cars.son, R. M., and E. Gutmann 1972. Development of contractile properties of minced muscle regenerates in the rat. Exp. Neurol 36:239-249.

5 Carlson, B. M., and E. Gutmann. 1975. Regeneration in free grafts of normal and denervated muscles in the rat: Morophology and listochemistry. Anat. Rec. 183: 47-62.

6. Carlson, B. M., and E. Gutmann. 1975. Regeneration in grafts of normal and denervated rat muscles: Contractile properties. Pfïgers Arch. 353: 215-225.

7. Carlson, B. M., and E. Gutmann. 1976. Contractile and histochemical properties of sliced muscle grafts regenerating in normal and denervated rat limbs. Exp. Neurol 50 : 319-329.

8. Guth, L., and F. J. Samaha. 1970. Procedure for the histochemical demonstration of actomyosin ATPase. Exp. Neurol. 28 : 365-367.

9. Gutmann, E., and B. M. Carlson. 1975. A comparison between the free grafting of sliced and intact muscles in the rat. Experientia $31: 848-849$.

10 Hall-CragGs, E. C. B. 1974. Rapid degeneration and regeneration of a whole skeletal muscle following treatment with bupivacaine (Marcaine). Exp. Neurol. 43 : 349-358.

11. JrRmanoví, I., and S. TheslefF. 1972. Ultrastructural study of experimental muscle degencration and regeneration in the adult rat. Z. Zellforsch. 131:77-97.

12. Nachlas, M. M., K. C. Tsou, E. Souza, C. S. Cheng, and A. M. Seligman. 1957. Cystochemical demonstration of succinc dehydrogenase by the use of a new p-nitrophenyl substituted ditetrazole. J. Histochem. Cytochem. 5: 420-436.

13. Padykula, H. A., and E. Herman. 1955. The specificity of the histochemical method for adenosine triphosphate. J Histochem Cytochem. 3: 170-195.

14. RoHliček, V., and E. Gutmann. 1972. The constant of contraction time, a new expression of maximal rate of tension development. Physiol. Bohemosloz. 21 : $430-431$. 